\title{
Insecticidal activity of the crude extract from Epichloë bromicola against Rhopalosiphum padi
}

\author{
Zhao-Ling Guo ${ }^{1}$, Fan $\mathrm{Li}^{1}$, Fang-Shu Cheng ${ }^{1}$ and Qiu-Yan Song ${ }^{1 *}$ \\ ${ }^{1}$ State Key Laboratory of Grassland Agro-ecosystems, Key Laboratory of Grassland Livestock Industry Innovation, Ministry of \\ Agriculture and Rural Affairs, College of Pastoral Agriculture Science and Technology, Lanzhou University, Lanzhou 730020, China
}

\begin{abstract}
Rhopalosiphum padi is a important pest in agricultural production. In order to reduce the use of chemical pesticides and improve the control effect, we reported the insecticidal activity of the crude extract from 20 strains Epichlö̈ bromicola isolated from Elymus tangutorum. The results showed that the crude extract of E. bromicola had certain insecticidal activity against $R$. padi. The experimental results showed that 10 strains with good activity were $2,3,5,7,8,11,16$, 18,12 and 19 , and their mortality rate reached more than $50 \%$ at $48 \mathrm{~h}$. However, the four strains of $13,14,15$ and 17 had no insecticidal activity, but could promote the growth of $R$. padi. Among them, strain 7 had the best insecticidal activity at 48h, and the mortality rate reached $88.3 \%$. The weakest insecticidal activity was strain 14 , with a mortality rate of $21.7 \%$. Strain 18 had the best insecticidal activity within $24 \mathrm{~h}$, with a mortality rate of $33.3 \%$. At the same time, the mortality rate of $48 \mathrm{~h}$ was significantly higher than that of $24 \mathrm{~h}$. In conclusion, these strains with significant insecticidal activity will be of great significance for the biological control of $R$. padi in the future.
\end{abstract}

\section{Introduction}

Rhopalosiphum padi is a kind of pest that has great influence on agricultural economy and is one of the main causes of crop yield and quality loss in horticulture, grain and trees ${ }^{[1,2]}$. $R$. padi sucked the juice of leaf blades, stems and young ears of plants with piercing-sucking mouthparts, resulting in yellowing and curling of the leaf blades (up to 90\%), poor plumpness of seeds, transmission of plant viruses, etc ${ }^{[3]}$. As a result, the wheat reduction reached $10 \%$ or above in China every year, especially in the outbreak year (up to $30 \%$ ). Therefore, $R$. padi was considered as one of the most important aphids bringing serious damage to wheat in China $^{[4]}$. It is estimated that aphids cause at least 2 per cent of all insect food loss to the world's crops each year. In addition to removing life fluids from plant screening elements, aphids are very effective vectors for viral diseases: about $60 \%$ of plant viruses are transmitted by these insects ${ }^{[5]}$. Various insecticides can be used to control aphids, but the extensive use of chemical insecticides has led to environmental pollution and the emergence of various aphid resistance populations ${ }^{[6]}$.

There is an urgent need for a biological pesticide to control $R$. padi. It has been reported that the secondary metabolites produced by E. bromicola are not toxic to normal animal cells and provide resistance to host plants ${ }^{[7,8]}$. Using this advantage, we studied the insect resistance of its crude extract.

In this paper, we used the same method to ferment $E$. bromicola at different altitudes to obtain crude extract, and tested the insecticidal activity of the E. bromicola against $R$. padi by leaf soaking method.

\section{Materials and methods}

These experiments were carried out at the Plant Protection Laboratory, Yuzhong Campus, Lanzhou University, Lanzhou, Gansu province.

\subsection{Instruments and equipment}

Rotary Evaporator (Tokyo Physiochemical Equipment Co.LTD, Tokyo, Japan); Hps-250 biochemical incubator (Harbin Donglian Electronic Technology Co.LTD, Harbin, China) Shaker incubator (Shanghai Zhichu Instrucment Co.LTD, Shanghai, China)

\subsection{The experimental materials}

\subsubsection{Fungal Strain}


The fungal strain used in this study was isolated from the Elymus tangutorum. The 20 strains isolated were kept in Institute of Grassland Agriculture Protection, Lanzhou University.

\subsubsection{Aphids}

The aphid in the experiment was $R$. padi and provided Institute of Plant Protection, Gansu Academy of Agricultural Sciences.

\subsection{General experimental procedures}

\subsubsection{Preparation of crude extract from E. bromicola}

Twenty strains of E. bromicola were cultured on potato dextrose agar (PDA) at $28{ }^{\circ} \mathrm{C}$ for 15 days as seed, respectively. Agar plugs were used to inoculate $1000-\mathrm{mL}$ Erlenmeyer flasks, each containing $400 \mathrm{~mL}$ of M104T (Sorbitol $100 \mathrm{~g}$, Glucose $40 \mathrm{~g}$, Yeast extract $3 \mathrm{~g}$, Glutamic acid $10 \mathrm{~g}$, Tryptophan $0.8 \mathrm{~g}, \mathrm{MgSO}_{4} .7 \mathrm{H}_{2} \mathrm{O} 0.3$ $\mathrm{g}, \mathrm{KH}_{2} \mathrm{PO}_{4} 1 \mathrm{~g}$ ). The flasks were placed on an incubatory shaker at $145 \mathrm{rpm}$ and $28{ }^{\circ} \mathrm{C}$ for 45 days. The fermentation broth of strain E. bromicola was separated into culture filtrate and mycelia by centrifugation. The culture and the mycelia were extracted with EtOAc (1.2 $\mathrm{mL}$ ) and $\mathrm{MeOH}(300 \mathrm{~mL})$ at room temperature (three times), respectively. Then, the EtOAc and $\mathrm{MeOH}$ were evaporated to dryness under vacuum on a rotar evaporator, respectively, and were combined to obtain a crude extract.

\subsubsection{Insecticidal Assay}

The insecticidal activity of crude extract against $R$. padi was evaluated by leaf-dip method using a previously reported procedure. The assay was repeated in an air-conditioned room at $25 \pm 2{ }^{\circ} \mathrm{C}$. Each tested sample was dissolved in acetone at a concentration of $2 \mathrm{~g} / \mathrm{L}$ and diluted with distilled water containing TW-80 $(0.1 \mathrm{mg} / \mathrm{L})$ to obtain a required concentration. Water containing TW-80 $(0.1 \mathrm{mg} / \mathrm{L})$ was used as control. Leaf disks $(5 \mathrm{~cm}$ $\times 3 \mathrm{~cm}$ ) were cut from fresh corn leaves and then dipped into the test solution for $6 \mathrm{~s}$. After air-drying the treated leaf disks and $30 \mathrm{R}$. padi were placed individually into the disks. The mortalities were evaluated $24 \mathrm{~h}$ and $48 \mathrm{~h}$ after treatment. All the assays were repeated in triplicate.

\subsubsection{Statistical analysis of data}

Excel was used to process the original test data and SPSS22.0 was used for significance difference analysis.

\section{Results and analysis}

The experimental results showed that the crude extract $E$. bromicola had certain insecticidal activity. The results showed that 10 strains had good insect resistance, which were $2,3,5,7,8,11,16,18,12$, and 19 , respectively.
The insecticidal activity of these strains was more significant than that of other strains, and the mortality rate of $48 \mathrm{~h}$ was more than $50 \%$.

\subsection{Treatment of $\boldsymbol{R}$. padi with crude extract for 24 hours}

The mortality rate of $R$. padi at $24 \mathrm{~h}$ was $33.3 \%$ and $6.67 \%$, respectively in strain 18 and strain 17 . Strain 17 had the same $6.67 \%$ as CK, so strain 17 had no inhibition on $R$. padi at $24 \mathrm{~h}$. The mortality rate of $R$. padi was $33.3 \%, 31.7 \%$, and $28.3 \%$, respectively in strain 18,11 and 19 .

According to Duncan's multiple comparison method, the difference of killing rate of 30 strains was not significant.

The $R$. padi mortality of 20 strains and CK $24 \mathrm{~h}$ ranged from large to small $(18>11>19>8>3=16>7=9=12=20>2>10>13>4>4>15=5>$ $6>\mathrm{CK}=17)$. Multiple comparison by Duncan's method found that there were significant differences among 17 , CK, 18 and 11. There is no difference between CK and 17; The difference of other treatments was not significant.

\subsection{Treatment of $R$. padi with crude extract for 48 hours}

The mortality rate of $R$. padi at $48 \mathrm{~h}$ was $88.3 \%$ and $21 \%$, $7 \%$, respectively in strain 7 and strain 14 . Strains 14,15 , and 17 had lower mortality rate than CK, so the three strains had the opposite effect and promoted aphid growth.

The mortality rate of $R$. padi was $88.3 \%, 75 \%$, and $71.7 \%$, respectively in strain 7,5 and 3 .

The $R$. padi mortality of 20 strains and CK $48 \mathrm{~h}$ ranged from large to small $(7>5>3>18>2>8>12=19>16>10>9=20>1>6>4>13>C K 1$ $7>15>14)$. According to Duncan's method, there were significant differences among $2,3,5,7,8,11,12,18,19$, and 14. There was no significant difference between 14 and other treatments.

\subsection{Comparison of $R$. padi mortality at $24 \mathrm{~h}$ and $48 \mathrm{~h}$}

As shown in Table 1., the mortality rate of $48 \mathrm{~h}$ was significantly higher than that of $24 \mathrm{~h}$. It can be seen from the Fig. 1. that the mortality of strains 3, 5, and 7 at $24 \mathrm{~h}$ and $48 \mathrm{~h}$ was significantly different, which was $46.7 \%$, $63.3 \%$ and $66.6 \%$, respectively. This indicates that these three strains will play a greater role after $24 \mathrm{~h}$, with more obvious insect resistance. The differences of strains 13, 14 , and 15 were small, $10 \%, 8.4 \%$ and $11.6 \%$, respectively. Compared with CK by $18.33 \%$, these three strains promoted the growth of $R$. padi between $24 \mathrm{~h}$ and $48 \mathrm{~h}$. 


\section{Conclusion}

In this paper, the insecticidal activity of 20 E. bromicola of Elymus tangutorum on $R$. padi was studied, and the time variables were set for $24 \mathrm{~h}$ and $48 \mathrm{~h}$. We found that $E$. bromicola were generally resistant to this $R$. padi and found the crude extract of strain 7 had the best insecticidal activity, up to $88.3 \%$.

Therefore, strain 7 can be used to inhibit $R$. padi. In the future, this crude extract can be further isolated and purified, and more effective pure compounds can be obtained to further improve the insecticidal activity.

Table 1. Mortality rate of $R$. padi at $24 \mathrm{~h}$ and $48 \mathrm{~h}$

\begin{tabular}{|l|l|l|l|}
\hline Strains & $\mathbf{2 4 h}(\boldsymbol{\%})$ & $\mathbf{4 8 h}(\boldsymbol{\%})$ & Difference (\%) \\
\hline CK & 6.67 & 25 & 18.33 \\
\hline 1 & 15 & 40 & 25 \\
\hline 2 & 20 & 65 & 45 \\
\hline 3 & 25 & 71.7 & 46.7 \\
\hline 4 & 15 & 31.7 & 16.7 \\
\hline 5 & 11.7 & 75 & 63.3 \\
\hline
\end{tabular}

\begin{tabular}{|l|l|l|l|}
\hline 6 & 10 & 36.7 & 26.7 \\
\hline 7 & 21.7 & 88.3 & 66.6 \\
\hline 8 & 26.7 & 60 & 33.3 \\
\hline 9 & 21.7 & 43.3 & 21.6 \\
\hline 10 & 18.3 & 46.7 & 28.4 \\
\hline 11 & 31.7 & 55 & 23.3 \\
\hline 12 & 21.7 & 53.3 & 31.6 \\
\hline 13 & 16.7 & 26.7 & 10 \\
\hline 14 & 13.3 & 21.7 & 8.4 \\
\hline 15 & 11.7 & 23.3 & 11.6 \\
\hline 16 & 25 & 51.7 & 26.7 \\
\hline 17 & 6.67 & 23.7 & 17.03 \\
\hline 18 & 33.3 & 70 & 36.7 \\
\hline
\end{tabular}

\section{Acknowledgement}

This project was supported financially by the National Natural Science Foundation of China (31901388), the Special Administrative Region (SAR) of Herbal Medicine in Gansu Province (18JR4RA003), the Fundamental Research Funds for the Central Universities (lzujbky-2020-20).

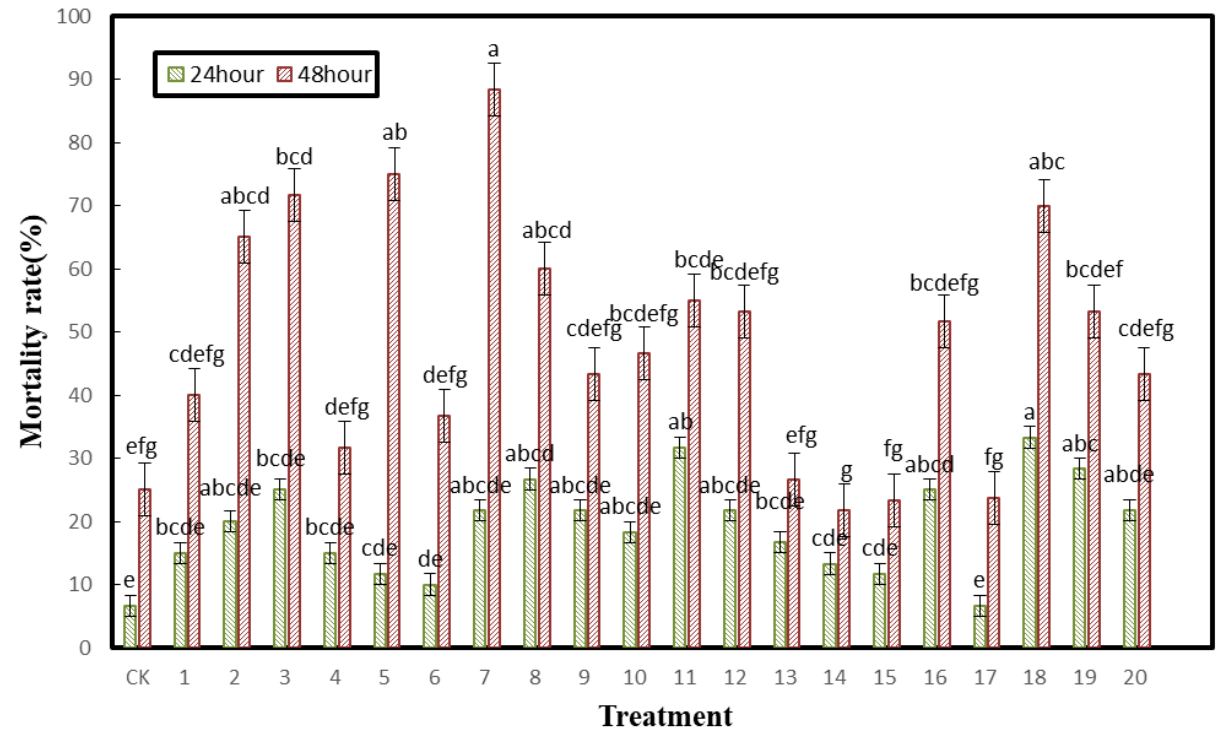

Fig. 1 Effects of E. bromicola on the insecticidal rate of R. padi

\section{References}

1. S. Ganassi, P. Grazioso, A. De Cristofaro, F. Fiorentini, M. A. Sabatini, A. Evidente, C. Altomare. Front Microbiol. Long Chain Alcohols Produced by Trichoderma citrinoviride Have Phagodeterrent Activity against the Bird Cherry-Oat Aphid Rhopalosiphum padi. 7, (2016)

2. Z. S. Batyrshina, B. Yaakov, R. Shavit, A. Singh, V. Tzin. Bmc Plant Biol. Comparative transcriptomic and metabolic analysis of wild and domesticated wheat genotypes reveals differences in chemical and physical defense responses against aphids. 20, (2020)

3. M. H. Chen, Z. J. Han, X. F. Qiao, M. J. Qu. Genome. Mutations in acetylcholinesterase genes of Rhopalosiphum padi resistant to organophosphate and carbamate insecticides. 50, 172-179 (2007)

4. L. Zhang, H. Lu, K. Guo, S. Yao, F. Cui. Sci China 
Life Sci. Insecticide resistance status and detoxification enzymes of wheat aphids Sitobion avenae and Rhopalosiphum padi. 60, 927-930 (2017)

5. A. Grudniewska, K. Dancewicz, A. Bialonska, C. Wawrzenczyk, B. Gabrys. J Agric Food Chem. Piperitone-derived saturated lactones: synthesis and aphid behavior-modifying activity. 61, 3364-3372 (2013)

6. F. Vanlerberghe-Masutti, T. Guillemaud. Biofutur. Resistance of aphids to insecticides. 27-30 (2007)
7. Q. Y. Song, Z. B. Nan, K. Gao, H. Song, P. Tian, X. X. Zhang, C. J. Li, W. B. Xu, X. Z. Li. J Agr Food Chem. Antifungal, Phytotoxic, and Cytotoxic Activities of Metabolites from Epichloe bromicola, a Fungus Obtained from Elymus tangutorum Grass. 63, 8787-8792 (2015)

8. T. Li, J. D. Blande, P. E. Gundel, M. Helander, K. Saikkonen. Plos One. Epichloe Endophytes Alter Inducible Indirect Defences in Host Grasses. 9, (2014) 